Discrete Comput Geom 27:551-565 (2002)

DOI: $10.1007 /$ s00454-001-0087-y

\title{
Toric Ideals and an Infinite Family of Normal (0, 1)-Polytopes without Unimodular Regular Triangulations*
}

\author{
Hidefumi Ohsugi \\ Department of Mathematics, Graduate School of Science, \\ Osaka University, Toyonaka, Osaka 560-0043, Japan \\ ohsugi@math.sci.osaka-u.ac.jp
}

\begin{abstract}
An infinite family of normal $(0,1)$-polytopes, none of whose unimodular triangulations is regular, is constructed. For the construction, we discuss triangulations of convex polytopes and flips of triangulations by studying markings on the set of circuits of toric ideals.
\end{abstract}

\section{Introduction}

Let $\mathcal{A}=\left\{\mathbf{a}_{1}, \ldots, \mathbf{a}_{n}\right\}$ be a finite subset of $\mathbb{Z}^{d}$ and suppose that $\mathcal{A}$ is contained in a hyperplane which does not contain the origin. Let $K$ be a field and let $K\left[\mathbf{t}, \mathbf{t}^{-1}\right]=$ $K\left[t_{1}, t_{1}^{-1}, \ldots, t_{d}, t_{d}^{-1}\right]$ be the Laurent polynomial ring in $d$ variables over $K$. Then we write $K[\mathcal{A}]$ for the subalgebra of $K\left[\mathbf{t}, \mathbf{t}^{-1}\right]$ which is generated by $\mathbf{t}^{\mathbf{a}_{1}}, \ldots, \mathbf{t}^{\mathbf{a}_{n}}$ over $K$. Here $\mathbf{t}^{\mathbf{a}_{i}}=\prod_{j=1}^{d} t_{j}^{\alpha_{j}}$ if $\mathbf{a}_{i}=\left(\alpha_{1}, \ldots, \alpha_{d}\right)$. Let $K[\mathbf{x}]=K\left[x_{1}, x_{2}, \ldots, x_{n}\right]$ denote the polynomial ring in $n$ variables over $K$ and let $\pi: K[\mathbf{x}] \rightarrow K[\mathcal{A}]$ be the surjective homomorphism of semigroup rings defined by $\pi\left(x_{i}\right)=\mathbf{t}^{\mathbf{a}_{i}}$ for all $1 \leq i \leq n$. We write $I_{\mathcal{A}}$ for the kernel of $\pi$ and call $I_{\mathcal{A}}$ the toric ideal of $\mathcal{A}$.

Let $\mathcal{P}_{\mathcal{A}}$ be the convex hull of $\mathcal{A}$. A triangulation $\Delta$ of $\mathcal{P}_{\mathcal{A}}$ is a geometric simplicial complex which covers $\mathcal{P}_{\mathcal{A}}$ and whose vertices belong to $\mathcal{A}$. The Stanley-Reisner ideal of $\Delta$ is a monomial ideal $I_{\Delta}=\left(x_{i_{1}} \cdots x_{i_{r}}: \operatorname{conv}\left(\mathbf{a}_{i_{1}}, \ldots, \mathbf{a}_{i_{r}}\right) \notin \Delta\right) \subset K[\mathbf{x}]$. Every generic vector $\omega \in \mathbb{R}^{n}$ defines a triangulation $\Delta_{\omega}$ as follows: The convex hull $\operatorname{conv}\left(\mathbf{a}_{i_{1}}, \ldots, \mathbf{a}_{i_{r}}\right)$ belongs to $\Delta_{\omega}$ if there exists a vector $\mathbf{c}=\left(c_{1}, \ldots, c_{d}\right) \in \mathbb{R}^{d}$ such that $\mathbf{a}_{j} \cdot \mathbf{c}=\omega_{j}$ if $j \in\left\{i_{1}, \ldots, i_{r}\right\}$ and $\mathbf{a}_{j} \cdot \mathbf{c}<\omega_{j}$ if $j \in\{1, \ldots, n\} \backslash\left\{i_{1}, \ldots, i_{r}\right\}$. A triangulation $\Delta$ of $\mathcal{P}_{\mathcal{A}}$ is called regular (or coherent) if $\Delta=\Delta_{\omega}$ for some $\omega \in \mathbb{R}^{n}$. If $\Delta$ is a regular triangulation,

* This research was supported by JSPS Research Fellowships for Young Scientists. 
then it is known [St, Theorem 8.3] that there exists a term order $\prec$ on $K[\mathbf{x}]$ such that the radical ideal of the initial ideal $\operatorname{in}_{\prec}\left(I_{\mathcal{A}}\right)$ of $I_{\mathcal{A}}$ is the Stanley-Reisner ideal $I_{\Delta}$ of $\Delta$.

A binomial $f \in I_{\mathcal{A}}$ is called a circuit if $f$ is irreducible and has minimal support. Here, the support of $f=\prod_{i=1}^{n} x_{i}^{p_{i}}-\prod_{j=1}^{n} x_{j}^{q_{j}}$ is defined by $\operatorname{supp}(f)=\left\{i: p_{i}>\right.$ 0 or $\left.q_{i}>0\right\}$. We also define the support of a monomial $m=\prod_{i=1}^{n} x_{i}^{p_{i}} \in K[\mathbf{x}]$ and a vector $\mathbf{v}=\left(v_{1}, \ldots, v_{n}\right) \in \mathbb{R}^{n}$ by $\operatorname{supp}(m)=\left\{i: p_{i}>0\right\}$ and $\operatorname{supp}(\mathbf{v})=\left\{i: v_{i} \neq 0\right\}$. Let $C_{\mathcal{A}}$ be the set of all circuits of $I_{\mathcal{A}}$. We consider a marking in(.) on $C_{\mathcal{A}}$, i.e., for each binomial $f \in C_{\mathcal{A}}$, in $(f)$ is one of the terms of $f$. We say that a marking in $(\cdot)$ is coherent if there exists a term order $\prec$ such that in $(f)=\operatorname{in}_{\prec}(f)$ for all $f \in C_{\mathcal{A}}$. In Section 1 we study the relation between triangulations and markings on $C_{\mathcal{A}}$. In particular, we study a criterion for markings on $C_{\mathcal{A}}$ to be associated with a triangulation. See Theorem 1.10. In Section 2 we discuss flips (or modifications or bistellar operations) of triangulations of $\mathcal{P}_{\mathcal{A}}$. Consult [D], [GKZ] and [R] for details about flips. It is known [GKZ] that any two regular triangulations are connected by a finite number of flips. On the other hand, recently, it turns out [Sa] that there exists a convex polytope having a (nonregular) triangulation which has NO flips. In Theorem 2.2 we give an algebraic approach to flips.

In Section 3 we apply these results to construct a desired infinite family of $(0,1)$ polytopes. A triangulation $\Delta$ of $\mathcal{P}_{\mathcal{A}}$ is called unimodular if the normalized volume of every maximal simplex in $\Delta$ equals one. A configuration $\mathcal{A}$ is called unimodular if all triangulations of $\mathcal{P}_{\mathcal{A}}$ are unimodular. The following six properties on a configuration $\mathcal{A}$ have been investigated in many papers on commutative algebra and combinatorics:

(i) $\mathcal{A}$ is unimodular;

(ii) $\mathcal{P}_{\mathcal{A}}$ is compressed, i.e., the regular triangulation with respect to any reverse lexicographic order is unimodular;

(iii) $\mathcal{P}_{\mathcal{A}}$ possesses a unimodular regular triangulation;

(iv) $\mathcal{P}_{\mathcal{A}}$ possesses a unimodular triangulation;

(v) $\mathcal{P}_{\mathcal{A}}$ possesses a unimodular covering;

(vi) $\mathcal{P}_{\mathcal{A}}$ is normal, i.e., the semigroup ring $K[\mathcal{A}]$ is normal.

There is the hierarchy (i) $\Rightarrow$ (ii) $\Rightarrow$ (iii) $\Rightarrow$ (iv) $\Rightarrow$ (v) $\Rightarrow$ (vi). However, the converse of each of the five implications is false. See [BG1], [BG2], [FZ] and [OH2]. Let $G$ be a finite connected graph having no loops and no multiple edges on the vertex set $V(G)=$ $\{1,2, \ldots, d\}$ and let $E(G)=\left\{e_{1}, e_{2}, \ldots, e_{n}\right\}$ be the set of edges of $G$. If $e=\{i, j\}$ is an edge of $G$ joining $i \in V(G)$ with $j \in V(G)$, then we define $\rho(e) \in \mathbb{R}^{d}$ by $\rho(e)=\mathbf{e}_{i}+\mathbf{e}_{j}$. Here $\mathbf{e}_{i}$ is the $i$ th unit coordinate vector in $\mathbb{R}^{d}$. Let $\mathcal{A}_{G}=\{\rho(e): e \in E(G)\}$. We set $\mathcal{P}_{G}$ for $\mathcal{P}_{\mathcal{A}_{G}}$ and call $\mathcal{P}_{G}$ the edge polytope of $G$. See also [OH1], [OH2] and [OH3] for edge polytopes.

Suppose that $G$ has a vertex $i_{0}$ of degree 2 . Then, we can construct a new graph $\hat{G}$ with $d+2$ vertices and $n+2$ edges by the following operation:

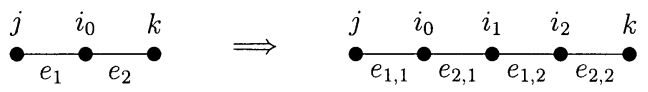

In [OH2] an edge polytope $\mathcal{P}_{G_{1}}$, none of whose regular triangulations is unimodular and having a unimodular triangulation obtained by one flip from a regular triangulation, was studied. Moreover, $\mathcal{P}_{G_{2}}$ in Example 3.2 is the second polytope which has the same 
property as $\mathcal{P}_{G_{1}}$. However, any other polytope which has the same property does not seem to be known so far. The graph $G_{1}$ has five vertices $v_{1}, \ldots, v_{5}$ of degree 2 . Let $G_{1}\left(p_{1}, p_{2}, \ldots, p_{5}\right)$ be the graph obtained from $G_{1}$ by applying the above operation $\left(p_{i}-1\right)$ times to the vertex $v_{i}$ for $1 \leq i \leq 5$. Similarly, from $G_{2}$, we can define the graph $G_{2}\left(q_{1}, q_{2}, \ldots, q_{12}\right)$ obtained the same way as above since $G_{2}$ has 12 vertices of degree 2. By the results in Sections 1 and 2 we obtain

Theorem 3.10. The edge polytopes $\mathcal{P}_{G_{1}\left(p_{1}, p_{2}, \ldots, p_{5}\right)}$ and $\mathcal{P}_{G_{2}\left(q_{1}, q_{2}, \ldots, q_{12}\right)}$ are normal $(0,1)$-polytopes, none of whose regular triangulations is unimodular and having a unimodular triangulation obtained by one flip from a regular triangulation.

\section{Triangulations and Markings on $C_{\mathcal{A}}$}

In this section we study the relation between triangulations and markings on circuits. Let $\mathcal{A}=\left\{\mathbf{a}_{1}, \ldots, \mathbf{a}_{n}\right\}$ be a finite subset of $\mathbb{Z}^{d}$ and suppose that $\mathcal{A}$ is contained in a hyperplane which does not contain the origin. We may assume that $\operatorname{rank}(\mathcal{A})=d$ if we regard $\mathcal{A}$ as a matrix. (If not, we can delete suitable rows of $\mathcal{A}$.) Let $K$ be a field and let $K\left[\mathbf{t}, \mathbf{t}^{-1}\right]=K\left[t_{1}, t_{1}^{-1}, \ldots, t_{d}, t_{d}^{-1}\right]$ be the Laurent polynomial ring in $d$ variables over $K$. Then we write $K[\mathcal{A}]$ for the subalgebra of $K\left[\mathbf{t}, \mathbf{t}^{-1}\right]$ which is generated by $\mathbf{t}^{\mathbf{a}_{1}}, \ldots, \mathbf{t}^{\mathbf{a}_{n}}$ over $K$. Here $\mathbf{t}^{\mathbf{a}_{i}}=\prod_{j=1}^{d} t_{j}^{\alpha_{j}}$ if $\mathbf{a}_{i}=\left(\alpha_{1}, \ldots, \alpha_{d}\right)$. Let $K[\mathbf{x}]=K\left[x_{1}, x_{2}, \ldots, x_{n}\right]$ denote the polynomial ring in $n$ variables over $K$ and let $\pi: K[\mathbf{x}] \rightarrow K[\mathcal{A}]$ denote the surjective homomorphism of semigroup rings defined by $\pi\left(x_{i}\right)=\mathbf{t}^{\mathbf{a}_{i}}$ for all $1 \leq i \leq n$. We write $I_{\mathcal{A}}$ for the kernel of $\pi$ and call $I_{\mathcal{A}}$ the toric ideal of $\mathcal{A}$. It is known that $I_{\mathcal{A}}$ is generated by homogeneous binomials. A binomial $f \in I_{\mathcal{A}}$ is called a circuit if $f$ is irreducible and has minimal support. Let $C_{\mathcal{A}}$ be the set of all circuits of $I_{\mathcal{A}}$. Now, we consider the marking $\operatorname{in}(\cdot)$ on $C_{\mathcal{A}}$, i.e., for each binomial $f \in C_{\mathcal{A}}$, in $(f)$ is one of the terms of $f$. We say that a marking in( $(\cdot)$ is coherent if there exists a term order $\prec$ such that in $(f)=\operatorname{in}_{\prec}(f)$ for all $f \in C_{\mathcal{A}}$. Let in $\left(C_{\mathcal{A}}\right)$ denote the monomial ideal (in $(f): f \in C_{\mathcal{A}}$ ).

A triangulation $\Delta$ of $\mathcal{P}_{\mathcal{A}}$ is called unimodular if the normalized volume of every maximal simplex in $\Delta$ equals one. Here the normalized volume of the maximal simplex $\sigma \in \Delta$ with the vertex set $V(\sigma)$ is defined by the index $[\mathbb{Z} \mathcal{A}: \mathbb{Z} \sigma]$ of the additive group $\mathbb{Z} \sigma=\sum_{\mathbf{a}_{i} \in V(\sigma)} \mathbb{Z} \mathbf{a}_{i}$ in $\mathbb{Z} \mathcal{A}=\sum_{i=1}^{n} \mathbb{Z} \mathbf{a}_{i}\left(\subset \mathbb{Z}^{d}\right)$. Moreover, $\mathcal{A}$ is called unimodular if all triangulations of $\mathcal{P}_{\mathcal{A}}$ are unimodular. It is known that $\mathcal{A}$ is unimodular if and only if both terms of any circuits of $I_{\mathcal{A}}$ are squarefree. See, e.g., Proposition 3.3 of [OHH].

The following three facts follow easily from Lemma 4.10 of [St].

Lemma 1.1. Let $0 \neq \mathbf{x}^{\mathbf{u}^{+}}-\mathbf{x}^{\mathbf{u}^{-}} \in I_{\mathcal{A}}$. Then there exists a circuit $\mathbf{x}^{\mathbf{v}^{+}}-\mathbf{x}^{\mathbf{v}^{-}} \in C_{\mathcal{A}}$ such that $\operatorname{supp}\left(\mathbf{v}^{+}\right) \subseteq \operatorname{supp}\left(\mathbf{u}^{+}\right)$and $\operatorname{supp}\left(\mathbf{v}^{-}\right) \subseteq \operatorname{supp}\left(\mathbf{u}^{-}\right)$.

Proposition 1.2. Let $0 \neq \mathbf{x}^{\mathbf{u}^{+}}-\mathbf{x}^{\mathbf{u}^{-}} \in I_{\mathcal{A}}$. Then, there exists a positive integer $m$ and a monomial $M \in K[\mathbf{x}]$ such that

$$
\left(\mathbf{x}^{\mathbf{u}^{+}}\right)^{m}-\left(\mathbf{x}^{\mathbf{u}^{-}}\right)^{m}=M\left(\prod_{i=1}^{p}\left(\mathbf{x}^{\mathbf{v}_{i}{ }^{+}}\right)^{m_{i}}-\prod_{i=1}^{p}\left(\mathbf{x}^{\mathbf{v}_{i}{ }^{-}}\right)^{m_{i}}\right),
$$


where each $m_{i} \in \mathbb{N}$, each $\mathbf{x}^{\mathbf{v}_{i}{ }^{+}}-\mathbf{x}^{\mathbf{v}_{i}{ }^{-}} \in C_{\mathcal{A}}$ and $1 \leq p \leq n-d$. In addition, if $\mathcal{A}$ is unimodular, then we have $m=1$.

Lemma 1.3. Let $I, J \subseteq\{1,2, \ldots, n\}$ with $I \neq J$ and suppose that the equation

$$
\sum_{i \in I} a_{i} \mathbf{a}_{i}=\sum_{j \in J} b_{j} \mathbf{a}_{j}
$$

holds, where $0<a_{i}, b_{j} \in \mathbf{Q}$. Then there exists a circuit $\mathbf{x}^{\mathbf{v}^{+}}-\mathbf{x}^{\mathbf{v}^{-}} \in C_{\mathcal{A}}$ such that $\operatorname{supp}\left(\mathbf{x}^{\mathbf{v}^{+}}\right) \subseteq I$ and $\operatorname{supp}\left(\mathbf{x}^{\mathbf{v}^{-}}\right) \subseteq J$.

The following result is derived from Theorem 8.3 of [St] and Remark 1.10 of [ST].

Proposition 1.4. Let $C_{\mathcal{A}}$ be the set of circuits of $I_{\mathcal{A}}$ and let $\prec$ be a term order. Then we have $\sqrt{\operatorname{in}_{\prec}\left(I_{\mathcal{A}}\right)}=\sqrt{\text { in }_{\prec}\left(C_{\mathcal{A}}\right)}$.

Hence, we can consider the set of circuits $C_{\mathcal{A}}$ instead of $I_{\mathcal{A}}$ in Theorem 8.3 of [St] to study triangulations of $\mathcal{P}_{\mathcal{A}}$. However, note that in ${ }_{\prec}\left(I_{\mathcal{A}}\right) \neq \mathrm{in}_{\prec}\left(C_{\mathcal{A}}\right)$, in general.

If $\sigma$ is a convex polytope, then let $V(\sigma)$ denote the set of all vertices of $\sigma$. We often identify $\left\{\mathbf{a}_{1}, \ldots, \mathbf{a}_{n}\right\}$ with $\{1, \ldots, n\}$. Thanks to Proposition 1.4 , the following theorem generalizes Theorem 8.3 of [St].

Theorem 1.5. Let $\Delta$ be a triangulation of $\mathcal{P}_{\mathcal{A}}$. Then there exists a marking in(.) on $C_{\mathcal{A}}$ such that $I_{\Delta}=\sqrt{\operatorname{in}\left(C_{\mathcal{A}}\right)}$. In particular, $\Delta$ is regular if and only if there exists a coherent marking in(.) on $C_{\mathcal{A}}$ such that $I_{\Delta}=\sqrt{\operatorname{in}\left(C_{\mathcal{A}}\right)}$.

Proof. Let a binomial $f=\mathbf{x}^{v^{+}}-\mathbf{x}^{v^{-}}$belong to $I_{\mathcal{A}}$. Then we have $\sum_{i \in \operatorname{supp}\left(v^{+}\right)} v_{i} \mathbf{a}_{i}=$ $\sum_{j \in \operatorname{supp}\left(v^{-}\right)} v_{j} \mathbf{a}_{j}$ where $0<v_{i} \in \mathbb{Z}$. Moreover, since $I_{\mathcal{A}}$ is homogeneous in the usual grading, we have $\sum_{i \in \operatorname{supp}\left(v^{+}\right)} v_{i}=\sum_{j \in \operatorname{supp}\left(v^{-}\right)} v_{j}$. Hence, two polytopes $\operatorname{conv}\left(\operatorname{supp}\left(v^{+}\right)\right)$ and $\operatorname{conv}\left(\operatorname{supp}\left(v^{-}\right)\right)$intersect in their interior. Since $\Delta$ is a triangulation, either $\operatorname{supp}\left(v^{+}\right)$ or $\operatorname{supp}\left(v^{-}\right)$is a nonface of $\Delta$, i.e., either $\mathbf{x}^{v^{+}}$or $\mathbf{x}^{v^{-}}$belongs to $I_{\Delta}$.

We consider a marking in(.) such that in $(f) \in I_{\Delta}$ for each $f \in C_{\mathcal{A}}$. (If all terms of $f$ belong to $I_{\Delta}$, then we can choose one of them for in $(f)$.) Suppose that $\sigma$ is a minimal nonface of $\Delta$. Now we choose a point $\sum_{\mathbf{a}_{i} \in V(\sigma)} a_{i} \mathbf{a}_{i} \in \sigma \subset \mathcal{P}_{\mathcal{A}}$ where $0<a_{i} \in \mathbf{Q}$ and $\sum_{\mathbf{a}_{i} \in V(\sigma)} a_{i}=1$. Since $\Delta$ is a triangulation, there exists a simplex $\sigma^{\prime} \in \Delta$ such that $\sum_{\mathbf{a}_{i} \in V(\sigma)} a_{i} \mathbf{a}_{i}=\sum_{\mathbf{a}_{j} \in V\left(\sigma^{\prime}\right)} b_{j} \mathbf{a}_{j}$ where $0<b_{j} \in \mathbf{Q}$ and $\sum_{\mathbf{a}_{j} \in \sigma^{\prime}} b_{j}=1$. Thanks to Lemma 1.3, there exists a circuit $f=\mathbf{x}^{v^{+}}-\mathbf{x}^{v^{-}} \in C_{\mathcal{A}}$ such that $\operatorname{supp}\left(v^{+}\right) \subseteq V(\sigma)$ and $\operatorname{supp}\left(v^{-}\right) \subseteq V\left(\sigma^{\prime}\right)$. Since $\sigma^{\prime} \in \Delta$, we have $\mathbf{x}^{v^{-}} \notin I_{\Delta}$ and $\mathbf{x}^{v^{+}} \in I_{\Delta}$. Hence, $\operatorname{supp}\left(v^{+}\right)=V(\sigma)$ and $\operatorname{in}(f)=\mathbf{x}^{v^{+}}$. Thus, we have $I_{\Delta}=\sqrt{\operatorname{in}\left(C_{\mathcal{A}}\right)}$. Moreover, by our Proposition 1.4 and Theorem 8.3 of [St], $\Delta$ is regular if and only if there exists a coherent marking in $(\cdot)$ on $C_{\mathcal{A}}$ such that $I_{\Delta}=\sqrt{\operatorname{in}\left(I_{\mathcal{A}}\right)}=\sqrt{\operatorname{in}\left(C_{\mathcal{A}}\right)}$.

The converse of Theorem 1.5 is false in general, i.e., there are markings in(.) such that $\sqrt{\operatorname{in}\left(C_{\mathcal{A}}\right)}$ is not the Stanley-Reisner ideal of any triangulation of $\mathcal{P}_{\mathcal{A}}$. 
Example 1.6. Let $\mathcal{A}=\{(1,0,1,0,0),(1,0,0,1,0),(1,0,0,0,1),(0,1,1,0,0)$, $(0,1,0,1,0),(0,1,0,0,1)\} \subset \mathbb{Z}^{5}$. Then $C_{\mathcal{A}}=\left\{f_{1}=x_{1} x_{5}-x_{2} x_{4}, f_{2}=x_{2} x_{6}-\right.$ $\left.x_{3} x_{5}, f_{3}=x_{3} x_{4}-x_{1} x_{6}\right\}$. Now we consider a noncoherent marking in(.) defined by $\operatorname{in}\left(f_{1}\right)=x_{1} x_{5}$, in $\left(f_{2}\right)=x_{2} x_{6}$ and in $\left(f_{3}\right)=x_{3} x_{4}$. Then $\sqrt{\operatorname{in}\left(C_{\mathcal{A}}\right)}=\left(x_{1} x_{5}, x_{2} x_{6}, x_{3} x_{4}\right)$. Suppose that there exists a triangulation $\Delta$ of $\mathcal{P}_{\mathcal{A}}$ with $I_{\Delta}=\sqrt{\operatorname{in}\left(C_{\mathcal{A}}\right)}$. Then sets of vertices of maximal simplices in $\Delta$ are $\left\{\mathbf{a}_{1}, \mathbf{a}_{2}, \mathbf{a}_{3}\right\},\left\{\mathbf{a}_{1}, \mathbf{a}_{2}, \mathbf{a}_{4}\right\},\left\{\mathbf{a}_{1}, \mathbf{a}_{3}, \mathbf{a}_{6}\right\},\left\{\mathbf{a}_{1}, \mathbf{a}_{4}, \mathbf{a}_{6}\right\}$, $\left\{\mathbf{a}_{2}, \mathbf{a}_{3}, \mathbf{a}_{5}\right\},\left\{\mathbf{a}_{2}, \mathbf{a}_{4}, \mathbf{a}_{5}\right\},\left\{\mathbf{a}_{3}, \mathbf{a}_{5}, \mathbf{a}_{6}\right\},\left\{\mathbf{a}_{4}, \mathbf{a}_{5}, \mathbf{a}_{6}\right\}$. Since $\operatorname{dim} \mathcal{P}_{\mathcal{A}}=3$, this is a contradiction. Thus, there exists no triangulation $\Delta$ of $\mathcal{P}_{\mathcal{A}}$ with $I_{\Delta}=\sqrt{\operatorname{in}\left(C_{\mathcal{A}}\right)}$.

However, note that, geometrically, $\Delta$ in Example 1.6 is a simplicial complex on the vertex set $\mathcal{A}$. Let $\Delta_{\text {in }}=\left\{\mathcal{P}_{\mathcal{B}} \subset \mathbb{R}^{d}: \mathcal{B} \subseteq \mathcal{A}, \prod_{\mathrm{a}_{i} \in \mathcal{B}} x_{i} \notin \sqrt{\operatorname{in}\left(C_{\mathcal{A}}\right)}\right\}$.

Proposition 1.7. Let in(.) be a marking on $C_{\mathcal{A}}$. Then $\Delta_{\text {in }}$ is a simplicial complex on the vertex set $\mathcal{A}$ such that $I_{\Delta_{\mathrm{in}}}=\sqrt{\operatorname{in}\left(C_{\mathcal{A}}\right)}$.

Proof. If $\sigma \in \Delta_{\text {in }}$ is not a subsimplex of $\mathcal{P}_{\mathcal{A}}$, then there exists an affine dependence on the vertices of $\sigma$, i.e., there exist two disjoint subsets $J_{1}$ and $J_{2}$ of $V(\sigma)$ such that

$$
\sum_{\mathbf{a}_{i} \in J_{1}} a_{i} \mathbf{a}_{i}=\sum_{\mathbf{a}_{j} \in J_{2}} b_{j} \mathbf{a}_{j},
$$

where $0<a_{i}, b_{j} \in \mathbf{Q}$ and $\sum_{\mathbf{a}_{i} \in J_{1}} a_{i}=\sum_{\mathbf{a}_{j} \in J_{2}} b_{j}=1$. Thanks to Lemma 1.3 there exists a circuit $f=\mathbf{x}^{v^{+}}-\mathbf{x}^{v^{-}} \in C_{\mathcal{A}}$ such that $\operatorname{supp}\left(v^{+}\right) \subseteq J_{1}$ and $\operatorname{supp}\left(v^{-}\right) \subseteq J_{2}$. Then neither $\mathbf{x}^{v^{+}}$nor $\mathbf{x}^{v^{-}}$belongs to $\sqrt{\operatorname{in}\left(C_{\mathcal{A}}\right)}$. This is a contradiction. Hence, $\sigma$ is a subsimplex of $\mathcal{P}_{\mathcal{A}}$.

Let $\sigma_{1}, \sigma_{2} \in \Delta_{\text {in }}$. We have to show that $\sigma_{1} \cap \sigma_{2}=\operatorname{conv}\left(V\left(\sigma_{1}\right) \cap V\left(\sigma_{2}\right)\right)$. We choose a point $\alpha \in \sigma_{1} \cap \sigma_{2}$. Then we have

$$
\alpha=\sum_{\mathbf{a}_{i} \in V\left(\sigma_{1}\right)} a_{i} \mathbf{a}_{i}=\sum_{\mathbf{a}_{j} \in V\left(\sigma_{2}\right)} b_{j} \mathbf{a}_{j},
$$

where $0 \leq a_{i}, b_{j} \in \mathbf{Q}$ and $\sum_{\mathbf{a}_{i} \in V\left(\sigma_{1}\right)} a_{i}=\sum_{\mathbf{a}_{j} \in V\left(\sigma_{2}\right)} b_{j}=1$. By multiplying the above equation by a suitable integer, we have

$$
\sum_{\mathbf{a}_{i} \in V\left(\sigma_{1}\right)} a_{i}^{\prime} \mathbf{a}_{i}=\sum_{\mathbf{a}_{j} \in V\left(\sigma_{2}\right)} b_{j}^{\prime} \mathbf{a}_{j}
$$

where $0 \leq a_{i}^{\prime}, b_{j}^{\prime} \in \mathbb{Z}$ and $\sum_{\mathbf{a}_{i} \in V\left(\sigma_{1}\right)} a_{i}^{\prime}=\sum_{\mathbf{a}_{j} \in V\left(\sigma_{2}\right)} b_{j}^{\prime}$. If $g=\prod_{\mathbf{a}_{i} \in V\left(\sigma_{1}\right)} x_{i}^{a_{i}^{\prime}}-$ $\prod_{\mathbf{a}_{j} \in V\left(\sigma_{2}\right)} x_{j}^{b_{j}^{\prime}} \neq 0$, then the homogeneous binomial $g$ belongs to $I_{\mathcal{A}}$. Thanks to Lemma 1.1 there exists a circuit $f=\mathbf{x}^{v^{+}}-\mathbf{x}^{v^{-}} \in C_{\mathcal{A}}$ such that $\operatorname{supp}\left(v^{+}\right) \subseteq V\left(\sigma_{1}\right)$ and $\operatorname{supp}\left(v^{-}\right) \subseteq$ $V\left(\sigma_{2}\right)$. Then neither $\mathbf{x}^{v^{+}}$nor $\mathbf{x}^{v^{-}}$belongs to $\sqrt{\operatorname{in}\left(C_{\mathcal{A}}\right)}$. This is a contradiction, and we have $g=0$. Hence, $a_{i}=0$ if $\mathbf{a}_{i} \notin V\left(\sigma_{1}\right) \cap V\left(\sigma_{2}\right)$. Then

$$
\alpha=\sum_{\mathbf{a}_{i} \in V\left(\sigma_{1}\right) \cap V\left(\sigma_{2}\right)} a_{i} \mathbf{a}_{i} .
$$

Thus, $\sigma_{1} \cap \sigma_{2} \subseteq \operatorname{conv}\left(V\left(\sigma_{1}\right) \cap V\left(\sigma_{2}\right)\right)$. Since the converse inclusion is trivial, $\Delta_{\text {in }}$ is a simplicial complex on the vertex set $\mathcal{A}$. 
If $\Delta_{\text {in }}$ is a triangulation of $\mathcal{P}_{\mathcal{A}}$, then we call in(.) a geometric marking on $C_{\mathcal{A}}$. For a marking in(.) on $C_{\mathcal{A}}$, we define the subsets $\mathcal{G}_{\text {in }}^{(1)}$ and $\mathcal{G}_{\text {in }}^{(2)}$ of $C_{\mathcal{A}}$ as follows:

$$
\begin{aligned}
& \mathcal{G}_{\text {in }}^{(1)}=\left\{f \in C_{\mathcal{A}}: f-\operatorname{in}(f) \notin \sqrt{\operatorname{in}\left(C_{\mathcal{A}}\right)}\right\}, \\
& \mathcal{G}_{\text {in }}^{(2)}=\left\{f \in \mathcal{G}_{\text {in }}^{(1)}: \text { there exists no } g \in C_{\mathcal{A}} \text { such that } \operatorname{supp}(\operatorname{in}(g)) \stackrel{\subsetneq}{\neq} \operatorname{supp}(\operatorname{in}(f))\right\} .
\end{aligned}
$$

Remark. Suppose that $\mathcal{A}$ is unimodular. Then it is known [St, Proposition 8.11] that every reduced Gröbner basis of $I_{\mathcal{A}}$ is contained in $C_{\mathcal{A}}$. Hence, if in(.) is a coherent marking (i.e., a term order on $K[\mathbf{x}]$ ), then $\mathcal{G}_{\text {in }}^{(2)}$ coincides with the reduced Gröbner basis of $I_{\mathcal{A}}$ with respect to in( $\left.\cdot\right)$.

Theorem 1.8. Suppose that $\operatorname{in}(\cdot)$ is a geometric marking on $C_{\mathcal{A}}$. Then we have $\sqrt{\operatorname{in}\left(\mathcal{G}_{\text {in }}^{(2)}\right)}=\sqrt{\operatorname{in}\left(C_{\mathcal{A}}\right)}$.

Proof. In the proof of Theorem 1.5, we have shown that if $\sigma$ is a minimal nonface of $\Delta_{\text {in }}$, then there exists a circuit $f=\mathbf{x}^{v^{+}}-\mathbf{x}^{v^{-}} \in C_{\mathcal{A}}$ such that $\operatorname{supp}\left(v^{+}\right)=V(\sigma)$ and $\mathbf{x}^{v^{-}} \notin \sqrt{\operatorname{in}\left(C_{\mathcal{A}}\right)}$. Since $f \in \mathcal{G}_{\text {in }}^{(2)}$, this completes the proof.

Corollary 1.9. Let in(.) and in'(.) be geometric markings on $C_{\mathcal{A}}$. Then $\Delta_{\mathrm{in}}=\Delta_{\mathrm{in}^{\prime}}$ if and only if $\mathcal{G}_{\mathrm{in}}^{(2)}=\mathcal{G}_{\mathrm{in}^{\prime}}^{(2)}$ and in $(g)=\mathrm{in}^{\prime}(g)$ for all $g \in \mathcal{G}_{\mathrm{in}}^{(2)}$.

Proof. Since $\mathcal{G}_{\text {in }}^{(2)}$ is uniquely determined by $\Delta_{\text {in }}$, the "only if" part holds. On the other hand, Theorem 1.8 enables us to show the "if" part.

Now we study a criterion for a marking on $C_{\mathcal{A}}$ to be a geometric marking. Note that, in conditions (ii) and (iii) below, $m^{\prime}$ is unique for each $\{m, p\},\left\{m, p^{\prime}\right\}$.

Theorem 1.10. For a marking in(.) on $C_{\mathcal{A}}$, the following conditions are equivalent:

(i) in(.) is a geometric marking;

(ii) there exists a positive integer $p$ and a monomial $m^{\prime} \notin \sqrt{\operatorname{in}\left(C_{\mathcal{A}}\right)}$ such that $m^{p}-m^{\prime} \in I_{\mathcal{A}}$ for an arbitrary monomial $m \in \operatorname{in}\left(C_{\mathcal{A}}\right)$;

(iii) there exists a positive integer $p^{\prime}$ and a sequence of reductions from $m^{p^{\prime}}$ to $m^{\prime} \notin \sqrt{\operatorname{in}\left(C_{\mathcal{A}}\right)}$ modulo $\mathcal{G}_{\text {in }}^{(1)}$ for an arbitrary monomial $m \in \operatorname{in}\left(C_{\mathcal{A}}\right)$.

Moreover, if $\mathcal{A}$ is unimodular, then we have $p=p^{\prime}=1$.

Proof. First, (iii) $\Rightarrow$ (ii) is trivial since $m^{p^{\prime}}-m^{\prime} \in I_{\mathcal{A}}$.

Second, we show that (ii) $\Rightarrow$ (iii). Suppose (ii). By virtue of Proposition 1.2, there exists a positive integer $k$ and a monomial $M \in K[\mathbf{x}]$ such that

$$
m^{p k}-\left(m^{\prime}\right)^{k}=M\left(\prod_{i=1}^{q}\left(\mathbf{x}^{\mathbf{v}_{i}{ }^{+}}\right)^{m_{i}}-\prod_{i=1}^{q}\left(\mathbf{x}^{\mathbf{v}_{i}{ }^{-}}\right)^{m_{i}}\right),
$$


where each $m_{i} \in \mathbb{N}$, each $\mathbf{x}^{\mathbf{v}_{i}{ }^{+}}-\mathbf{x}^{\mathbf{v}_{i}{ }^{-}} \in C_{\mathcal{A}}$. Since $m^{\prime} \notin \sqrt{\operatorname{in}\left(C_{\mathcal{A}}\right)}$, we have $\mathbf{x}^{\mathbf{v}_{i}{ }^{-}} \notin$ $\sqrt{\operatorname{in}\left(C_{\mathcal{A}}\right)}$ and hence each $\mathbf{x}^{\mathbf{v}_{i}{ }^{+}}-\mathbf{x}^{\mathbf{v}_{i}{ }^{-}}$belongs to $\mathcal{G}_{\text {in }}^{(1)}$. Thus, there exists a reduction from $m^{p k}$ to $\left(m^{\prime}\right)^{k} \notin \sqrt{\text { in }\left(C_{\mathcal{A}}\right)}$ modulo $\mathcal{G}_{\text {in }}^{(1)}$.

Third, we show that (i) $\Rightarrow$ (ii). Suppose that in(.) on $C_{\mathcal{A}}$ is a geometric marking. Let $m=\prod_{i=1}^{n} x_{i}^{a_{i}} \in \operatorname{in}\left(C_{\mathcal{A}}\right), s=\sum_{i=1}^{n} a_{i}$ and $a_{i}^{\prime}=a_{i} / s$. Then we have $\alpha=\sum_{i \in \operatorname{supp}(m)} a_{i}^{\prime} \mathbf{a}_{i} \in \mathcal{P}_{\mathcal{A}}$ since $0<a_{i}^{\prime} \in \mathbf{Q}$ and $\sum_{i \in \operatorname{supp}(m)} a_{i}^{\prime}=1$. Since $\Delta_{\text {in }}$ is a triangulation, there exists a unique simplex $\sigma \in \Delta_{\text {in }}$ such that $\alpha=\sum_{i \in \operatorname{supp}(m)} a_{i}^{\prime} \mathbf{a}_{i}=$ $\sum_{\mathbf{a}_{j} \in V(\sigma)} b_{j} \mathbf{a}_{j}$, where $0<b_{j} \in \mathbf{Q}$ and $\sum_{\mathbf{a}_{j} \in V(\sigma)} b_{j}=1$. Then there exists a positive integer $p$ such that $f=m^{p}-\prod_{\mathbf{a}_{j} \in V(\sigma)} x_{j}^{b_{j} s p} \in I_{\mathcal{A}}$. Since $\sigma \in \Delta$, we have $\prod_{\mathbf{a}_{j} \in V(\sigma)} x_{j}^{b_{j} s p} \notin \sqrt{\operatorname{in}\left(C_{\mathcal{A}}\right)}$.

Suppose that $\mathcal{A}$ is unimodular. Since $s \cdot \alpha \in \mathbb{Z} \mathcal{A}$ and $\sigma$ is a face of a simplex of normalized volume 1 , we have $b_{j} s \in \mathbb{N}$ for all $j$ with $\mathbf{a}_{j} \in V(\sigma)$. Hence, we have $f^{\prime}=m-\prod_{\mathbf{a}_{j} \in V(\sigma)} x_{j}^{b_{j} s} \in I_{\mathcal{A}}$ and $\prod_{\mathbf{a}_{j} \in V(\sigma)} x_{j}^{b_{j} s} \notin \sqrt{\operatorname{in}\left(C_{\mathcal{A}}\right)}$.

Finally, we show that (ii) $\Rightarrow$ (i). Thanks to Proposition 1.7, in order to show (i), what we must prove is $\mathcal{P}_{\mathcal{A}}=\bigcup_{\sigma \in \Delta_{\text {in }}} \sigma$. Let $\alpha=\sum_{i=1}^{n} a_{i} \mathbf{a}_{i} \in \mathcal{P}_{\mathcal{A}} \backslash \bigcup_{\sigma \in \Delta_{\text {in }}} \sigma$ where $0 \leq a_{i} \in \mathbf{Q}$ and $\sum_{i=1}^{n} a_{i}=1$. By multiplying a suitable integer $z$, we have $z \cdot \alpha=\sum_{i=1}^{n} a_{i}^{\prime} \mathbf{a}_{i}$ where $0 \leq a_{i}^{\prime} \in \mathbb{Z}$. Now we consider the monomial $m=\prod_{i=1}^{n} x_{i}^{a_{i}^{\prime}}$. Since $\alpha \notin \bigcup_{\sigma \in \Delta_{\text {in }}} \sigma$, we have $m \in \sqrt{\operatorname{in}\left(C_{\mathcal{A}}\right)}$. By the hypothesis, there exists a positive integer $p$ and a monomial $m^{\prime}=\prod_{j \in J} x_{j}^{b_{j}} \notin \sqrt{\operatorname{in}\left(C_{\mathcal{A}}\right)}$ such that $m^{p}-m^{\prime} \in I_{\mathcal{A}}$. Hence,

$$
\alpha=\sum_{i=1}^{n} a_{i} \mathbf{a}_{i}=\sum_{j \in J}\left(\frac{b_{j}}{z p}\right) \mathbf{a}_{j} .
$$

Since $m^{\prime} \notin \sqrt{\operatorname{in}\left(C_{\mathcal{A}}\right)}$, we have $J \in \Delta_{\text {in }}$. This is a contradiction, and we have $\mathcal{P}_{\mathcal{A}}=$ $\bigcup_{\sigma \in \Delta_{\text {in }}} \sigma$ as desired.

\section{Flips and Markings on $C_{\mathcal{A}}$}

Let $\Delta$ be a triangulation of $\mathcal{P}_{\mathcal{A}}$ and let $f=\mathbf{x}^{u^{+}}-\mathbf{x}^{u^{-}} \in C_{\mathcal{A}}$ be a circuit. We say that $\Delta$ is supported on $f$ if the following two conditions are satisfied:

(i) $\operatorname{conv}(\operatorname{supp}(f) \backslash\{j\}) \in \Delta$ for all $j \in \operatorname{supp}\left(\mathbf{x}^{u^{+}}\right)$.

(ii) Let $F_{1}=\operatorname{supp}(f) \backslash\left\{j_{1}\right\}$ and $F_{2}=\operatorname{supp}(f) \backslash\left\{j_{2}\right\}$ where $j_{1}, j_{2} \in \operatorname{supp}\left(\mathbf{x}^{u^{+}}\right)$. Then, for every subset $F \subseteq \mathcal{A} \backslash \operatorname{supp}(f), \operatorname{conv}\left(F_{1} \cup F\right)$ belongs to $\Delta$ if and only if $\operatorname{conv}\left(F_{2} \cup F\right)$ belongs to $\Delta$.

The above condition (ii) is the usual link condition in Definition 2.9 of Chapter 7 of [GKZ]. We can rewrite (i) and (ii) as follows:

(i) $\prod_{i \in \operatorname{supp}(f) \backslash\{j\}} x_{i} \notin I_{\Delta}$ for all $j \in \operatorname{supp}\left(\mathbf{x}^{u^{+}}\right)$.

(ii) For all monomials $m \in K[\mathbf{x}] \operatorname{such}$ that $\operatorname{supp}(m) \cap \operatorname{supp}(f)=\emptyset$ and for all $j_{1}, j_{2} \in \operatorname{supp}\left(\mathbf{x}^{u^{+}}\right)$, we have $m \cdot \prod_{i \in \operatorname{supp}(f) \backslash\left\{j_{1}\right\}} x_{i}$ belongs to $I_{\Delta}$ if and only if $m \cdot \prod_{i \in \operatorname{supp}(f) \backslash\left\{j_{2}\right\}} x_{i}$ belongs to $I_{\Delta}$.

If a triangulation $\Delta$ of $\mathcal{P}_{\mathcal{A}}$ is supported on a circuit $f \in C_{\mathcal{A}}$, then we can construct a new triangulation by taking away all the simplices of the form $\operatorname{supp}(m) \cup \operatorname{supp}(f) \backslash\{i\}$ where 
$i \in \operatorname{supp}\left(\mathbf{x}^{u^{+}}\right)$and $m \in K[\mathbf{x}]$ is a monomial with $\operatorname{supp}(m) \cap \operatorname{supp}(f)=\emptyset$ and adding the simplices of the form $\operatorname{supp}(m) \cup \operatorname{supp}(f) \backslash\{j\}$ where $j \in \operatorname{supp}\left(\mathbf{x}^{u^{-}}\right)$and the same $m$. We call this operation a flip along $f$. See [GKZ] for details. Note that flips among monomial $\mathcal{A}$-graded ideals are studied in [MT]. For every monomial $\mathcal{A}$-graded ideal $I$, there exists a triangulation $\Delta$ of $\mathcal{P}_{\mathcal{A}}$ with $I_{\Delta}=\sqrt{I}$. However, the converse is false in general.

First, we represent a flip of a triangulation $\Delta$ of $\mathcal{P}_{\mathcal{A}}$ as an operation for a geometric marking in $(\cdot)$ such that $\Delta=\Delta_{\text {in }}$. Suppose that a triangulation $\Delta_{\text {in }}$ is supported on a circuit $f \in C_{\mathcal{A}}$ and $\Delta^{\prime}$ is obtained by a flip from $\Delta_{\text {in }}$ along $f$. Now we define markings in $^{*}(\cdot)$ and in' $(\cdot)$ on $C_{\mathcal{A}}$ by

$$
\begin{aligned}
\operatorname{in}^{*}(g) & =\left\{\begin{array}{ll}
g-\operatorname{in}(g), & g \notin \mathcal{G}_{\text {in }}^{(1)} \\
\operatorname{in}(g), & \text { otherwise, }
\end{array} \text { and } \quad \operatorname{supp}(\operatorname{in}(f)) \subseteq \operatorname{supp}(\operatorname{in}(g)),\right. \\
\operatorname{in}^{\prime}(g) & =\left\{\begin{array}{ll}
g-\operatorname{in}(g), & g=f, \\
g-\operatorname{in}(g), & g \notin \mathcal{G}_{\text {in }}^{(1)} \\
\operatorname{in}(g), & \text { otherwise. }
\end{array} \text { and } \quad \operatorname{supp}(\operatorname{in}(f)) \subseteq \operatorname{supp}(\operatorname{in}(g)),\right.
\end{aligned}
$$

By the definition, $f$ belongs to $\mathcal{G}_{\text {in }}^{(1)}$ if $\Delta_{\text {in }}$ is supported on $f$. Hence we have

$$
\operatorname{in}^{\prime}(g)= \begin{cases}g-\operatorname{in}^{*}(g), & g=f, \\ \operatorname{in}^{*}(g), & \text { otherwise. }\end{cases}
$$

Theorem 2.1. Work with the same situation as above. Then we have $\Delta_{\mathrm{in}}=\Delta_{\mathrm{in}^{*}}$ and $\Delta^{\prime}=\Delta_{\text {in' }}$.

Proof. First, thanks to Theorem 1.8, we have $\sqrt{\operatorname{in}\left(C_{\mathcal{A}}\right)}=\sqrt{\text { in }^{*}\left(C_{\mathcal{A}}\right)}$. Hence, we have $\Delta_{\text {in }}=\Delta_{\text {in }^{*}}$.

For a monomial $m$, if $m \in I_{\Delta}$ and if $m \notin I_{\Delta^{\prime}}$, then $\operatorname{supp}(\operatorname{in}(f)) \subseteq \operatorname{supp}(m)$. Similarly, if $m \notin I_{\Delta}$ and if $m \in I_{\Delta^{\prime}}$, then $\operatorname{supp}(f-\operatorname{in}(f)) \subseteq \operatorname{supp}(m)$. Suppose that there exists a circuit $g \in C_{\mathcal{A}}$ such that in' $(g) \notin I_{\Delta^{\prime}}$. It then follows that $g-\operatorname{in}^{\prime}(g) \in I_{\Delta^{\prime}}$. By the definition of flips, we have in' $(f)=f-\operatorname{in}(f) \in I_{\Delta^{\prime}}$. Hence, we have $g \neq f$. Suppose that $g \in \mathcal{G}_{\text {in }}^{(1)}$. By the definition (1), in' $(g)=\operatorname{in}(g) \in I_{\Delta}$. Hence, we have supp(in $\left.(f)\right) \subseteq \operatorname{supp}\left(\operatorname{in}^{\prime}(g)\right)$. Moreover, since $g-\operatorname{in}^{\prime}(g) \notin I_{\Delta}$, we have $\operatorname{supp}(f-\operatorname{in}(f)) \subseteq \operatorname{supp}\left(g-\operatorname{in}^{\prime}(g)\right)$. This contradicts that $g$ is a circuit. Suppose that $g \notin \mathcal{G}_{\text {in }}^{(1)}$, i.e., both $\operatorname{in}(g)$ and $g-\operatorname{in}(g)$ belong to $I_{\Delta}$. Since in' $(g) \notin I_{\Delta^{\prime}}$, we have $\operatorname{supp}(\operatorname{in}(f)) \subseteq \operatorname{supp}\left(\operatorname{in}^{\prime}(g)\right)$. If in' $(g)=$ $\operatorname{in}(g)$, then $\operatorname{supp}(\operatorname{in}(f)) \subseteq \operatorname{supp}\left(\operatorname{in}^{\prime}(g)\right)=\operatorname{supp}(\operatorname{in}(g))$. This contradicts the definition (1). If $\operatorname{in}^{\prime}(g)=g-\operatorname{in}(g)$, then we have supp(in $\left.(f)\right) \subseteq \operatorname{supp}(\operatorname{in}(g))$ by the definition (1). Since $\operatorname{supp}(\operatorname{in}(f)) \subseteq \operatorname{supp}(g-\operatorname{in}(g))$ and $\operatorname{supp}(\operatorname{in}(f)) \subseteq \operatorname{supp}(\operatorname{in}(g))$, this contradicts that $g$ is a circuit. Hence, we have $\sqrt{\operatorname{in}^{\prime}\left(C_{\mathcal{A}}\right)} \subseteq I_{\Delta^{\prime}}$. Thus, $\Delta^{\prime} \subseteq \Delta_{\text {in }^{\prime}}$. Thanks to Proposition 1.7 and since $\Delta^{\prime}$ is a triangulation, we have $\Delta^{\prime}=\Delta_{\mathrm{in}^{\prime}}$ as required.

Now we want to know which circuits support a triangulation. In the statement of Theorem 2.2, similar form appears as in the $S$-polynomial $S(f, g)$ of the binomials 


$$
\begin{gathered}
f=\mathbf{x}^{u^{+}}-\mathbf{x}^{u^{-}} \in I_{\mathcal{A}} \text { and } g=\mathbf{x}^{v^{+}}-\mathbf{x}^{v^{-}} \in I_{\mathcal{A}} \text { with } \operatorname{in}(f)=\mathbf{x}^{u^{+}} \text {and } \operatorname{in}(g)=\mathbf{x}^{v^{+}}: \\
S(f, g)=\frac{\operatorname{LCM}(\operatorname{in}(f), \operatorname{in}(g))}{\operatorname{in}(f)} \cdot \mathbf{x}^{u^{-}}-\frac{\operatorname{LCM}(\operatorname{in}(f), \operatorname{in}(g))}{\operatorname{in}(g)} \cdot \mathbf{x}^{v^{-}} .
\end{gathered}
$$

Theorem 2.2. Let in(.) be a geometric marking and let $f=\mathbf{x}^{u^{+}}-\mathbf{x}^{u^{-}} \in C_{\mathcal{A}}$ with $\operatorname{in}(f)=\mathbf{x}^{u^{+}}$. If $p=\max \left(\left|u_{i}\right| \in \mathbb{Z}: \mathbf{x}^{u^{+}}-\mathbf{x}^{u^{-}} \in C_{\mathcal{A}}\right)$, then a triangulation $\Delta_{\operatorname{in}}$ of $\mathcal{P}_{\mathcal{A}}$ is supported on $f$ if and only if $f \in \mathcal{G}_{\mathrm{in}}^{(2)}$ and

$$
\frac{\operatorname{LCM}\left(\operatorname{in}(f)^{p}, \operatorname{in}(g)\right)}{\operatorname{in}(f)^{p}} \cdot \mathbf{x}^{u^{-}} \in \sqrt{\operatorname{in}\left(C_{\mathcal{A}}\right)}
$$

for all $g \in \mathcal{G}_{\text {in }}^{(2)} \backslash\{f\}$. Moreover, if $\mathcal{A}$ is unimodular, we have $p=1$.

Proof. [only if] Suppose that $\Delta_{\text {in }}$ is supported on a circuit $f=\mathbf{x}^{u^{+}}-\mathbf{x}^{u^{-}} \in C_{\mathcal{A}}$. Then $\prod_{i \in \operatorname{supp}(f) \backslash\{j\}} x_{i} \notin \sqrt{\operatorname{in}\left(C_{\mathcal{A}}\right)}$ for all $j \in \operatorname{supp}\left(\mathbf{x}^{u^{+}}\right)$. Hence, in particular, neither $\mathbf{x}^{u^{-}}$nor $\prod_{i \in \operatorname{supp}\left(\mathbf{x}^{u^{+}}\right) \backslash\{j\}} x_{i}$ belongs to $\sqrt{\operatorname{in}\left(C_{\mathcal{A}}\right)}$. Thus, $f \in \mathcal{G}_{\text {in }}^{(2)}$.

Suppose that

$$
M=\frac{\operatorname{LCM}\left(\operatorname{in}(f)^{p}, \operatorname{in}(g)\right)}{\operatorname{in}(f)^{p}} \cdot \mathbf{x}^{u^{-}} \notin \sqrt{\operatorname{in}\left(C_{\mathcal{A}}\right)}
$$

for a circuit $g=\mathbf{x}^{v^{+}}-\mathbf{x}^{v^{-}} \in \mathcal{G}_{\text {in }}^{(2)} \backslash\{f\}$ with in $(g)=\mathbf{x}^{v^{+}}$. Note that, by the definition of $p$, we have $\operatorname{supp}(M)=\left(\operatorname{supp}(\operatorname{in}(g)) \cup \operatorname{supp}\left(\mathbf{x}^{u^{-}}\right)\right) \backslash \operatorname{supp}(\operatorname{in}(f))$.

If $\operatorname{supp}\left(\mathbf{x}^{u^{+}}\right) \cap \operatorname{supp}\left(\mathbf{x}^{v^{+}}\right)=\emptyset$, then we have $M=\operatorname{in}(g) \cdot \mathbf{x}^{u^{-}} \in \operatorname{in}\left(C_{\mathcal{A}}\right)$. Hence, $\operatorname{supp}\left(\mathbf{x}^{u^{+}}\right) \cap \operatorname{supp}\left(\mathbf{x}^{v^{+}}\right) \neq \emptyset$. Suppose that $\operatorname{supp}\left(\mathbf{x}^{u^{+}}\right)=\operatorname{supp}\left(\mathbf{x}^{v^{+}}\right)$. Let $v_{r} / u_{r}=\min \left(v_{i} /\right.$ $\left.u_{i}>0: i \in \operatorname{supp}\left(\mathbf{x}^{u^{+}}\right)\right)$where $r \in \operatorname{supp}\left(\mathbf{x}^{u^{+}}\right)$. It then follows that $\left(\mathbf{x}^{u^{+}}\right)^{v_{r}} \operatorname{divides}\left(\mathbf{x}^{v^{+}}\right)^{u_{r}}$. Hence,

$$
0 \neq\left(\mathbf{x}^{v^{+}}\right)^{u_{r}}-\left(\mathbf{x}^{v^{-}}\right)^{u_{r}}-\frac{\left(\mathbf{x}^{v^{+}}\right)^{u_{r}}}{\left(\mathbf{x}^{u^{+}}\right)^{v_{r}}}\left(\left(\mathbf{x}^{u^{+}}\right)^{v_{r}}-\left(\mathbf{x}^{u^{-}}\right)^{v_{r}}\right)=\frac{\left(\mathbf{x}^{v^{+}}\right)^{u_{r}}}{\left(\mathbf{x}^{u^{+}}\right)^{v_{r}}} \cdot\left(\mathbf{x}^{u^{-}}\right)^{v_{r}}-\left(\mathbf{x}^{v^{-}}\right)^{u_{r}}
$$

belongs to $I_{\mathcal{A}}$. Since $\left(\mathbf{x}^{v^{-}}\right)^{u_{r}} \notin \sqrt{\operatorname{in}\left(C_{\mathcal{A}}\right)}$, we have $m=\left(\mathbf{x}^{v^{+}}\right)^{u_{r}} /\left(\mathbf{x}^{u^{+}}\right)^{v_{r}} \cdot\left(\mathbf{x}^{u^{-}}\right)^{v_{r}} \in$ $\sqrt{\operatorname{in}\left(C_{\mathcal{A}}\right)}$. Since $\operatorname{supp}(m) \subseteq \operatorname{supp}(f) \backslash\{r\}, \Delta_{\text {in }}$ is not supported on $f$.

Suppose that $\operatorname{supp}\left(\mathbf{x}^{u^{+}}\right) \neq \operatorname{supp}\left(\mathbf{x}^{v^{+}}\right)$and $\Delta^{\prime}$ is obtained by a flip from $\Delta_{\text {in }}$ along $f$. Since $g \in \mathcal{G}_{\text {in }}^{(2)}, \operatorname{supp}\left(\mathbf{x}^{u^{+}}\right) \backslash \operatorname{supp}\left(\mathbf{x}^{v^{+}}\right) \neq \emptyset$. If $i \in \operatorname{supp}\left(\mathbf{x}^{u^{+}}\right) \backslash \operatorname{supp}\left(\mathbf{x}^{v^{+}}\right)$, then $F=(\operatorname{supp}(\operatorname{in}(g)) \cup \operatorname{supp}(f)) \backslash\{i\}$ is a nonface of $\Delta_{\text {in }} \operatorname{since} \operatorname{supp}(\operatorname{in}(g)) \subseteq F$. Since $\operatorname{supp}(M)=F \backslash \operatorname{supp}(\operatorname{in}(f))$ and $\operatorname{supp}(M)$ is a face of $\Delta_{\text {in }}, \operatorname{supp}(M)$ is a face of $\Delta^{\prime}$ by the definition of flips. Hence, we have

$$
\frac{\operatorname{LCM}\left(\operatorname{in}(f)^{p}, \operatorname{in}(g)\right)}{\operatorname{in}(f)^{p}} \cdot \mathbf{x}^{u^{-}} \notin I_{\Delta^{\prime}} .
$$

Thus, $\mathbf{x}^{u^{-}} \notin I_{\Delta^{\prime}}$ and this contradicts Theorem 2.1.

[if] Suppose that $m=\prod_{i \in \operatorname{supp}(f) \backslash\{j\}} x_{i} \in \sqrt{\operatorname{in}\left(C_{\mathcal{A}}\right)}$ for some $j \in \operatorname{supp}\left(\mathbf{x}^{u^{+}}\right)$. Then there exists a circuit $g \in \mathcal{G}_{\text {in }}^{(2)}$ such that $\operatorname{supp}(\operatorname{in}(g)) \subseteq \operatorname{supp}(m)$. Since $\operatorname{supp}(\operatorname{in}(g)) \subseteq$ $\operatorname{supp}(f)$, we have

$$
\operatorname{supp}\left(\frac{\operatorname{LCM}\left(\operatorname{in}(f)^{p}, \operatorname{in}(g)\right)}{\operatorname{in}(f)^{p}} \cdot \mathbf{x}^{u^{-}}\right)=\operatorname{supp}\left(\mathbf{x}^{u^{-}}\right) \in \Delta_{\text {in }} .
$$


Suppose that there exists a (squarefree) monomial $m$ with $\operatorname{supp}(m) \cap \operatorname{supp}(f)=\emptyset$ such that $m_{1}=m \cdot \prod_{i \in \operatorname{supp}(f) \backslash\left\{j_{1}\right\}} x_{i} \notin \sqrt{\operatorname{in}\left(C_{\mathcal{A}}\right)}$ and $m_{2}=m \cdot \prod_{i \in \operatorname{supp}(f) \backslash\left\{j_{2}\right\}} x_{i} \in$ $\sqrt{\operatorname{in}\left(C_{\mathcal{A}}\right)}$ where $j_{1}, j_{2} \in \operatorname{supp}\left(\mathbf{x}^{u^{+}}\right)$. Then there exists a circuit $g \in \mathcal{G}_{\text {in }}^{(2)}$ such that $\operatorname{supp}(\operatorname{in}(g)) \subseteq \operatorname{supp}\left(m_{2}\right)$. Since $\operatorname{supp}\left(m_{2}\right) \backslash \operatorname{supp}(\operatorname{in}(f))=\operatorname{supp}\left(m \cdot \mathbf{x}^{u^{-}}\right)$, we have

$$
\begin{aligned}
\operatorname{supp}\left(\frac{\operatorname{LCM}\left(\operatorname{in}(f)^{p}, \operatorname{in}(g)\right)}{\operatorname{in}(f)^{p}} \cdot \mathbf{x}^{u^{-}}\right) & \subseteq \operatorname{supp}\left(\frac{\operatorname{LCM}\left(\operatorname{in}(f)^{p}, m_{2}\right)}{\operatorname{in}(f)^{p}} \cdot \mathbf{x}^{u^{-}}\right) \\
& =\operatorname{supp}\left(m \cdot \mathbf{x}^{u^{-}}\right) \subseteq \operatorname{supp}\left(m_{1}\right) \in \Delta_{\mathrm{in}}
\end{aligned}
$$

\section{An Application for Edge Polytopes}

Let $G$ be a finite connected graph having no loops and no multiple edges on the vertex set $V(G)=\{1,2, \ldots, d\}$ and let $E(G)=\left\{e_{1}, e_{2}, \ldots, e_{n}\right\}$ be the set of edges of $G$. If $e=\{i, j\}$ is an edge of $G$ joining $i \in V(G)$ with $j \in V(G)$, then we define $\rho(e) \in \mathbb{R}^{d}$ by $\rho(e)=\mathbf{e}_{i}+\mathbf{e}_{j}$. Here $\mathbf{e}_{i}$ is the $i$ th unit coordinate vector in $\mathbb{R}^{d}$. Let $\mathcal{A}_{G}=\{\rho(e): e \in E(G)\}$. We set $\mathcal{P}_{G}$ for $\mathcal{P}_{\mathcal{A}_{G}}$ and call $\mathcal{P}_{G}$ the edge polytope of $G$. We set $K[G]$ for $K\left[\mathcal{A}_{G}\right]$ and call $K[G]$ the edge ring of $G$ and set $I_{G}$ for $I_{\mathcal{A}_{G}}$ and call $I_{G}$ the toric ideal of $G$. In general, $\mathcal{P}_{\mathcal{A}}$ is called normal if $K[\mathcal{A}]$ is a normal semigroup ring. See also [OH1], [OH2] and [OH3] for edge polytopes. It is known [OH1, Corollary 2.3] that the following three conditions are equivalent: (i) $\mathcal{P}_{G}$ has a unimodular covering; (ii) $\mathcal{P}_{G}$ is normal; (iii) $G$ satisfies the odd cycle condition, i.e., for every two odd cycles without a common vertex, there is an edge of $G$ connecting two odd cycles.

First, we give two examples of graphs whose edge polytope is a normal polytope having a unimodular triangulation and having no regular unimodular triangulation.

Example 3.1. In [OH2] we give the following graph $G_{1}$ with 10 vertices and 15 edges. Then $\mathcal{P}_{G_{1}}$ is a normal $(0,1)$-polytope, none of whose regular triangulations is unimodular and having a unimodular nonregular triangulation obtained by one flip from a regular triangulation.

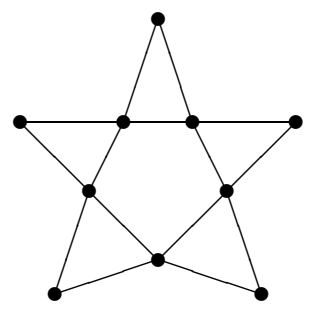

Since the graph $G_{1}$ given in Example 3.1 has 10 vertices and 15 edges $(n-d=5)$, its edge polytope is of dimension 9 with 15 vertices. The parameter $n-d$ is important for polytopes in general. (Note that the operation for graphs which will be defined later in this section preserves $n-d$.) If $n-d \leq 2$, then all triangulation of $\mathcal{P}_{\mathcal{A}}$ is regular ([L]). If $n-d \leq 3$, then all triangulation of $\mathcal{P}_{\mathcal{A}}$ is connected by flips ([AS]). By using the criterion [O, Theorem 3.5], it is not difficult to show that if a graph $G$ satisfying the 
odd cycle condition has $d$ vertices and $n$ edges with $n-d \leq 3$, then the edge polytope $\mathcal{P}_{G}$ possesses a regular unimodular triangulation. Thus, it is reasonable to ask if a graph $G$ satisfying the odd cycle condition has $d$ vertices and $n$ edges with $n-d \leq 4$, then the edge polytope $\mathcal{P}_{G}$ possesses a regular unimodular triangulation. However, there exists a graph below having 20 vertices and 24 edges whose edge polytope possesses no regular unimodular triangulation:

Example 3.2. This edge polytope was constructed by Ohsugi and Takayuki Hibi in 1997. Let $G_{2}$ be the following graph with 20 vertices and 24 edges:

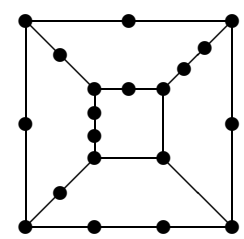

Then $\operatorname{dim} \mathcal{P}_{G_{2}}=19$ and the normalized volume of $\mathcal{P}_{G_{2}}$ is equal to 307 . There are three pairs $\left(C_{1}, C_{1}{ }^{\prime}\right),\left(C_{2}, C_{2}{ }^{\prime}\right),\left(C_{3}, C_{3}{ }^{\prime}\right)$ of two minimal odd cycles in $G_{2}$ having no common vertex. Each $\left(C_{i}, C_{i}{ }^{\prime}\right)$ has exactly one bridge $b_{i}$ and the even closed walk $\Gamma_{i}=\left(b_{i}, C_{i}, b_{i}, C_{i}{ }^{\prime}\right)$ satisfies the conditions in Proposition 3.3. By virtue of Lemma 3.3 of [OH3] or the technique of a combinatorial pure subring [OHH], we can show that each $f_{\Gamma_{i}}$ appears in the reduced Gröbner basis of $I_{G}$ with respect to any term order. Suppose that $I_{G}$ has a squarefree initial ideal $\operatorname{in}_{\succ}\left(I_{G}\right)$. Since one of the terms of each $f_{\Gamma_{i}}$ is not squarefree, $\succ$ satisfies that

$$
\left\{\begin{array}{l}
x_{1} x_{3} x_{4} x_{6} x_{8} x_{10} x_{11} x_{13} x_{15} \succ x_{2} x_{5} x_{7} x_{9} x_{12} x_{14} x_{16} x_{19}^{2}, \\
x_{2} x_{7} x_{10} x_{12} x_{17} x_{19} x_{20} x_{22} x_{24} \succ x_{1} x_{3} x_{6} x_{13} x_{18} x_{21} x_{23} x_{11}^{2}, \\
x_{5} x_{9} x_{11} x_{14} x_{16} x_{18} x_{19} x_{21} x_{23} \succ x_{4} x_{8} x_{15} x_{17} x_{20} x_{22} x_{24} x_{10}^{2}
\end{array} .\right.
$$

Since $\prod_{i=1}^{3} \operatorname{in}_{\succ}\left(f_{\Gamma_{i}}\right)=\prod_{i=1}^{3}\left(f_{\Gamma_{i}}-\operatorname{in}_{\succ}\left(f_{\Gamma_{i}}\right)\right)$, this contradicts that $\succ$ is a term order. Thus, with respect to any term order, the initial ideal of $I_{G_{2}}$ is not squarefree. Thanks to Corollary 8.9 of $[\mathrm{St}], \mathcal{P}_{G_{2}}$ has no regular unimodular triangulation. On the other hand, Firla and Ziegler [FZ, Example 12] verified that $\mathcal{P}_{G_{2}}$ does have a nonregular unimodular triangulation $\Delta_{2}$. Moreover, Imai verified that $\mathcal{P}_{G_{2}}$ has a nonregular unimodular triangulation obtained by one flip from a regular triangulation.

The main purpose of the present section is to give an infinite family of normal edge polytopes having the same property as $\mathcal{P}_{G_{1}}$ and $\mathcal{P}_{G_{2}}$.

Suppose that $G$ has a vertex $i_{0}$ of degree 2 . Then we can construct a new graph $\hat{G}$ with $d+2$ vertices and $n+2$ edges by the following operation:

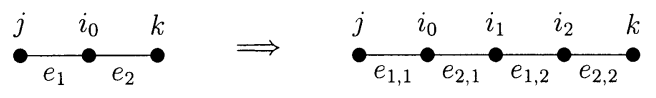

Then, thanks to Corollary 2.3 of [OH1], Proposition 3.3 follows. 
Proposition 3.3. Then $K[G]$ is normal if and only if $K[\hat{G}]$ is normal.

Let $K[\hat{\mathbf{x}}]=K\left[x_{1,1}, x_{1,2}, x_{2,1}, x_{2,2}, x_{3}, \ldots, x_{n}\right]$ denote the polynomial ring in $n+2$ variables over $K$. Now we define the injective homomorphism $\psi: K[\mathbf{x}] \rightarrow K[\hat{\mathbf{x}}]$ by

$$
\psi\left(x_{i}\right)= \begin{cases}x_{i, 1} x_{i, 2} & \text { if } \quad i=1,2, \\ x_{i} & \text { otherwise. }\end{cases}
$$

The set of all circuits of $I_{G}$ is explicitly classified. Given an even closed walk $\Gamma=$ $\left(e_{i_{1}}, e_{i_{2}}, \ldots, e_{i_{2 q}}\right)$ of $G$ with each $e_{k} \in E(G)$, we write $f_{\Gamma}$ for the binomial $f_{\Gamma}=$ $\prod_{k=1}^{q} x_{i_{2 k-1}}-\prod_{k=1}^{q} x_{i_{2 k}} \in I_{G}$. It is known that $I_{G}$ is generated by such $f_{\Gamma}$ 's. See Lemma 1.1 of [OH3]. Let $C_{G}$ denote the set of all circuits of $I_{G}$. Then the following is known [St, Lemma 9.8]:

Proposition 3.4. Let $G$ be a finite connected graph. Then $C_{G}$ consists of the binomials $f_{\Gamma}$ where $\Gamma$ is an even closed walk satisfying one of the following conditions:

(i) $\Gamma$ is an even cycle.

(ii) $\Gamma$ consists of two odd cycles having exactly one common vertex.

(iii) $\Gamma$ consists of two odd cycles having no common vertex and connected by a path.

Corollary 3.5. Let $G$ be a finite connected graph. Then the set $C_{\hat{G}}$ of all circuits equals $\left\{\psi(f) \in K[\hat{\mathbf{x}}]: f \in C_{G}\right\}$.

Proof. Let $\Gamma$ be an even closed walk of $G$ with $f_{\Gamma} \in C_{G}$. If $\Gamma$ contains neither $e_{1}$ nor $e_{2}$, then we have $\psi\left(f_{\Gamma}\right)=f_{\Gamma} \in C_{\hat{G}}$. Thanks to Proposition 3.4, if $\Gamma$ contains one of $e_{1}$ and $e_{2}$, then we have either $\Gamma=\left(e_{1}, e_{2}, e_{i_{1}}, \ldots, e_{i_{2 p}}\right)$ or $\Gamma=\left(e_{1}, e_{2}, e_{j_{1}}, \ldots, e_{j_{2 q+1}}, e_{1}, e_{2}, e_{k_{1}}\right.$, $\left.\ldots, e_{k_{2 r+1}}\right)$ where each of $i_{s}, j_{t}$ and $k_{u}$ does not belong to $\{1,2\}$. It then follows that $\Gamma^{\prime}=$ $\left(e_{1,1}, e_{2,1}, e_{1,2}, e_{2,2}, e_{i_{1}}, \ldots, e_{i_{2 p}}\right)$ or $\Gamma^{\prime}=\left(e_{1,1}, e_{2,1}, e_{1,2}, e_{2,2}, e_{j_{1}}, \ldots, e_{j_{2 q+1}}, e_{1,1}, e_{2,1}\right.$, $\left.e_{1,2}, e_{2,2}, e_{k_{1}}, \ldots, e_{k_{2 r+1}}\right)$ is an even closed walk of $\hat{G}$ satisfying one of the conditions in Proposition 3.4. Thus, $\psi\left(f_{\Gamma}\right)=f_{\Gamma^{\prime}} \in C_{\hat{G}}$. Conversely, let $\Gamma^{\prime}$ be an even closed walk of $\hat{G}$ with $f_{\Gamma^{\prime}} \in C_{\hat{G}}$. By the same way as above, we can find an even closed walk $\Gamma$ of $G$ satisfying $\psi\left(f_{\Gamma}\right)=f_{\Gamma^{\prime}}$ and $f_{\Gamma} \in C_{G}$ as required.

If in(.) is a marking on $C_{G}$, then we define the marking $\operatorname{In}(\cdot)$ on $C_{\hat{G}}$ by $\operatorname{In}(\psi(f))=$ $\psi(\operatorname{in}(f))$ for all $f \in C_{G}$. Thanks to Corollary 3.5 , this is a one-to-one correspondence between the set of all markings on $C_{G}$ and the set of all markings on $C_{\hat{G}}$.

Lemma 3.6. A marking in(.) on $C_{G}$ is a geometric marking if and only if corresponding marking $\operatorname{In}(\cdot)$ on $C_{\hat{G}}$ is a geometric marking.

Proof. Let $m$ be a monomial in $K[\mathbf{x}]$. Note that $m \in \sqrt{\operatorname{in}\left(C_{G}\right)}$ if and only if $\psi(m) \in$ $\sqrt{\operatorname{In}\left(C_{\hat{G}}\right)}$. Hence, $\psi\left(\mathcal{G}_{\text {in }}^{(i)}\right)=\mathcal{G}_{\text {In }}^{(i)}$ for $i=1,2$. It then follows that there exists a sequence of reductions from $m^{p}$ to $m^{\prime} \notin \sqrt{\operatorname{in}\left(C_{G}\right)}$ modulo $\mathcal{G}_{\text {in }}^{(1)}$ for a positive integer $p$ if and only if there exists a sequence of reductions from $(\psi(m))^{p}$ to $\psi\left(m^{\prime}\right) \notin \sqrt{\operatorname{In}\left(C_{\hat{G}}\right)}$ modulo $\mathcal{G}_{\text {In }}^{(1)}$. Thanks to Theorem 1.10, this completes the proof. 
Lemma 3.7. Let in(.) and $\operatorname{in}^{\prime}(\cdot)$ be geometric markings on $C_{G}$. Then $\Delta_{\mathrm{in}}=\Delta_{\mathrm{in}^{\prime}}$ if and only if $\Delta_{\mathrm{In}}=\Delta_{\mathrm{In}^{\prime}}$.

Proof. Since $\psi\left(\mathcal{G}_{\text {in }}^{(2)}\right)=\mathcal{G}_{\text {In }}^{(2)}$, Corollary 1.9 enable us to complete the proof.

We define a map $\bar{\psi}$ from the set of all triangulations of $\mathcal{P}_{G}$ to the set of all triangulations of $\mathcal{P}_{\hat{G}}$ by $\bar{\psi}\left(\Delta_{\text {in }}\right)=\Delta_{\text {In }}$ where in(.) is a geometric marking on $C_{G}$.

Theorem 3.8. The map $\bar{\psi}$ is a bijection from the set of all triangulations of $\mathcal{P}_{G}$ to the set of all triangulations of $\mathcal{P}_{\hat{G}}$. Moreover, if $\Delta$ is a triangulation of $\mathcal{P}_{G}$, then $\Delta$ is regular (resp. unimodular) if and only if $\bar{\psi}(\Delta)$ is regular (resp. unimodular).

Proof. Thanks to Lemmas 3.6 and 3.7, $\bar{\psi}$ is a bijection from the set of all triangulations of $\mathcal{P}_{G}$ to the set of all triangulations of $\mathcal{P}_{\hat{G}}$.

If in (.) on $C_{G}$ is coherent, then there is a weight vector $\left(\omega_{1}, \omega_{2}, \ldots, \omega_{n}\right) \in \mathbb{R}^{n}$ which represents in $(\cdot)$. Then $\left(\omega_{1} / 2, \omega_{1} / 2, \omega_{2} / 2, \omega_{2} / 2, \omega_{3}, \ldots, \omega_{n}\right) \in \mathbb{R}^{n+2}$ is a weight vector for $\operatorname{In}(\cdot)$ on $C_{\hat{G}}$. If $\operatorname{In}(\cdot)$ on $C_{\hat{G}}$ is coherent, then there is a weight vector $\left(\omega_{1,1}, \omega_{1,2}, \omega_{2,1}\right.$, $\left.\omega_{2,2}, \ldots, \omega_{n}\right) \in \mathbb{R}^{n+2}$ which represents $\operatorname{In}(\cdot)$. Then $\left(\omega_{1,1}+\omega_{1,2}, \omega_{2,1}+\omega_{2,2}, \omega_{3}, \ldots, \omega_{n}\right)$ $\in \mathbb{R}^{n}$ is a weight vector for in(.) on $C_{G}$. Hence, in(.) on $C_{G}$ is coherent if and only if $\operatorname{In}(\cdot)$ on $C_{\hat{G}}$ is coherent. Thanks to Theorem $1.5, \Delta$ is regular if and only if $\bar{\psi}(\Delta)$ is regular.

Since $I_{\Delta_{\text {in }}}=\bigcap_{\sigma \in \Delta_{\text {in }}}\left(x_{i}: \mathbf{a}_{i} \notin V(\sigma)\right)$, we have $I_{\Delta_{\mathrm{In}}}=\bigcap_{\sigma \in \Delta_{\text {in }}}\left(\psi\left(x_{i}\right): \mathbf{a}_{i} \notin V(\sigma)\right)$. Let $\sigma$ be a maximal simplex in $\Delta_{\text {in }}$. Since $\operatorname{dim}(\sigma)=d-1$, the graph associated with $\sigma$ is a spanning graph of $G$ by Lemma 9.5 of [St]. Since the degree of the vertex $v_{0}$ equals two, either $\mathbf{a}_{1} \in V(\sigma)$ or $\mathbf{a}_{2} \in V(\sigma)$. Hence, we have

$\left(\psi\left(x_{i}\right): \mathbf{a}_{i} \notin V(\sigma)\right)= \begin{cases}\left(x_{i}: \mathbf{a}_{i} \notin V(\sigma)\right) & \text { if } \quad \mathbf{a}_{1}, \mathbf{a}_{2} \in V(\sigma), \\ \bigcap_{j=1}^{2}\left(\left\{x_{1, j}\right\} \cup\left\{x_{i}: i \neq 1, \mathbf{a}_{i} \notin V(\sigma)\right\}\right) & \text { if } \quad \mathbf{a}_{1} \notin V(\sigma), \\ \bigcap_{j=1}^{2}\left(\left\{x_{2, j}\right\} \cup\left\{x_{i}: i \neq 2, \mathbf{a}_{i} \notin V(\sigma)\right\}\right) & \text { if } \quad \mathbf{a}_{2} \notin V(\sigma) .\end{cases}$

It then follows that $\bar{\psi}$ preserves the number of odd cycles in the subgraph of $G$ associated with a maximal simplex $\sigma \in \Delta_{\text {in }}$. By virtue of Lemma 9.5 of [St], this implies that $\bar{\psi}$ preserves the normalized volume of $\sigma \in \Delta_{\text {in }}$. Hence, $\Delta_{\text {in }}$ is unimodular if and only if $\bar{\psi}\left(\Delta_{\text {in }}\right)$ is unimodular as desired.

Theorem 3.9. Let $\Delta_{1}$ and $\Delta_{2}$ be triangulations of $\mathcal{P}_{G}$. Then $\Delta_{1}$ is obtained by the flip from $\Delta_{2}$ along the circuit $\Gamma$ if and only if $\bar{\psi}\left(\Delta_{1}\right)$ is obtained by the flip from $\bar{\psi}\left(\Delta_{2}\right)$ along the circuit $\psi(\Gamma)$.

Proof. Let $f, g \in \mathcal{G}_{\text {in }}^{(2)}$, let $p$ be a positive integer and let $m_{1}=\frac{\operatorname{LCM}\left(\operatorname{in}(f)^{p}, \operatorname{in}(g)\right)}{\operatorname{in}(f)^{p}} \cdot \mathbf{x}^{u^{-}} \quad$ and $\quad m_{2}=\frac{\operatorname{LCM}\left(\operatorname{In}(\psi(f))^{p}, \operatorname{In}(\psi(g))\right)}{\operatorname{In}(\psi(f))^{p}} \cdot \psi\left(\mathbf{x}^{u^{-}}\right)$. 
Since $\psi\left(m_{1}\right)=m_{2}, m_{1} \in \sqrt{\operatorname{in}\left(C_{G}\right)}$ if and only if $m_{2} \in \sqrt{\operatorname{In}\left(C_{\hat{G}}\right)}$. Hence, thanks to Theorem 2.2, $\Delta_{i}$ is supported on $\Gamma$ if and only if $\bar{\psi}\left(\Delta_{i}\right)$ is supported on $\psi(\Gamma)$. Since $\psi\left(\mathcal{G}_{\text {in }}^{(1)}\right)=\mathcal{G}_{\text {In }}^{(1)}$ and since $\operatorname{supp}(\operatorname{in}(f)) \subseteq \operatorname{supp}(\operatorname{in}(h))$ if and only if $\operatorname{supp}(\operatorname{In}(\psi(f))) \subseteq$ $\operatorname{supp}(\operatorname{In}(\psi(h)))$ for $h \in C_{G}$, Theorem 2.1 completes the proof.

Since $G_{1}$ in Example 3.1 has five vertices $v_{1}, v_{2}, \ldots, v_{5}$ of degree 2, we get an infinite family of normal edge polytopes having the same property as $\mathcal{P}_{G_{1}}$. Let $G_{1}\left(p_{1}, p_{2}, \ldots\right.$, $\left.p_{5}\right)$ be the graph obtained from $G_{1}$ by applying the operation $\left(p_{i}-1\right)$ times to the vertex $v_{i}$ for $1 \leq i \leq 5$. Each $G_{1}\left(p_{1}, p_{2}, \ldots, p_{5}\right)$ has $2 \sum_{i=1}^{5} p_{i}$ vertices and $5+2 \sum_{i=1}^{5} p_{i}$ edges. For example, $G_{1}(2,1,2,2,1)$ is the following graph:

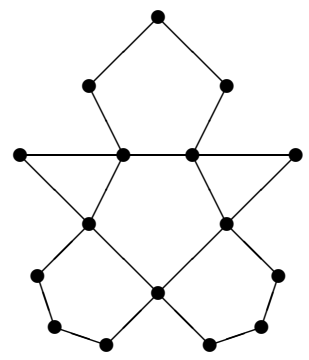

Similarly, from $G_{2}$ in Example 3.2, we can define the graph $G_{2}\left(q_{1}, q_{2}, \ldots, q_{12}\right)$ obtained in the same way as above since $G_{2}$ has 12 vertices of degree 2 .

Thanks to Theorems 3.8 and 3.9, we have the following theorem:

Theorem 3.10. The edge polytopes $\mathcal{P}_{G_{1}\left(p_{1}, p_{2}, \ldots, p_{5}\right)}$ and $\mathcal{P}_{G_{2}\left(q_{1}, q_{2}, \ldots, q_{12}\right)}$ are normal $(0,1)$-polytopes, none of whose regular triangulations is unimodular and having a unimodular triangulation obtained by one flip from a regular triangulation.

Finally, we give a normal edge polytope having no regular unimodular triangulation, having a unimodular triangulation and NONE of whose unimodular triangulation is obtained by one flip from a regular triangulation.

Example 3.11. Let $G_{3}$ be the following graph with 10 vertices and 15 edges:

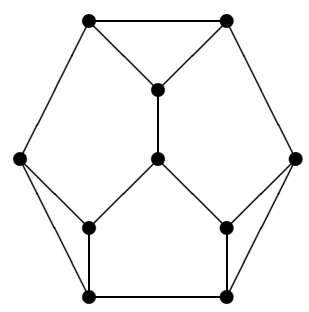

By the same technique appearing in Example 3.2, we can see that $\mathcal{P}_{G_{3}}$ has no regular unimodular triangulation. On the other hand, by explicit computation by PUNTOS, it is verified that $\mathcal{P}_{G_{3}}$ has a (nonregular) unimodular triangulation $\Delta_{3}$ and, moreover, $\mathcal{P}_{G_{3}}$ is the first polytope having a unimodular triangulation, none of whose unimodular 
triangulation is obtained by one flip from a regular triangulation. However, it is also verified by PUNTOS that $\Delta_{3}$ is obtained by two flips from a regular triangulation.

We do not know if there exists an edge polytope satisfying odd cycle condition which has no unimodular triangulation so far.

Conjecture 3.12. Let $G$ be a finite connected graph satisfying the odd cycle condition. Then $\mathcal{P}_{G}$ has a unimodular triangulation obtained by a finite number of flips from a regular triangulation.

\section{References}

[AS] M. Azaola and F. Santos, The graph of triangulations of a point configuration with $d+4$ vertices is 3-connected, Discrete Comput. Geom. 23 (2000), 489-536.

[BG1] C. Bouvier and G. Gonzalez-Sprinberg, Système générateur minimal, diviseurs essentiels et $G$ désingularisations de variétés toriques, Tôhoku Math. J. 46 (1994), 125-149.

[BG2] W. Bruns and J. Gubeladze, Normality and covering properties of affine semigroups, J. Reine Angew. Math. 510 (1999), 161-178.

[D] J. A. de Loera, Triangulations of polytopes and computational algebra, Ph.D. dissertation, Cornell University, 1995.

[FZ] R. T. Firla and G. M. Ziegler, Hilbert bases, unimodular triangulations, and binary covers of rational polyhedral cones, Discrete Comput. Geom. 21 (1999), 205-216.

[GKZ] I. M. Gelfand, M. M. Kapranov and A. V. Zelevinsky, Discriminants, Resultants and Multidimensional Determinants, Birkhäuser, Boston, 1994.

[L] C. W. Lee, Regular triangulations of convex polytopes, in Applied Geometry and Discrete Mathematics-The Victor Klee Festschrift (P. Gritzmann and B. Sturmfels, eds.), DIMACS Series 4, American Mathematical Society, Providence, RI, pp. 443-456.

[MT] D. Maclagan and R. R. Thomas, Combinatorics of the toric Hilbert scheme, Discrete Comput. Geom. 27 (2002), 249-264.

[O] H. Ohsugi, Unimodular regular triangulations of $(0,1)$-polytopes associated with finite graphs, in Algebraic Engineering (C. L. Nehaniv and M. Ito, eds.), World Scientific, Singapore, 1999, pp. 159171.

[OHH] H. Ohsugi, J. Herzog and T. Hibi, Combinatorial pure subrings, Osaka J. Math. 37 (2000), 745-757.

[OH1] H. Ohsugi and T. Hibi, Normal polytopes arising from finite graphs, J. Algebra 207 (1998), 409-426.

[OH2] H. Ohsugi and T. Hibi, A normal $(0,1)$-polytope none of whose regular triangulations is unimodular, Discrete Comput. Geom. 21 (1999), 201-204.

[OH3] H. Ohsugi and T. Hibi, Toric ideals generated by quadratic binomials, J. Algebra 218 (1999), 509-527.

[R] V. Reiner, The generalized Baues problem, in New Perspectives in Algebraic Combinatorics (L. Billera, A. Björner, R. Simion and R. Stanley, eds.), Cambridge University Press, Cambridge, 1999, pp. 293 336.

[Sa] F. Santos, A point configuration whose space of triangulations is disconnected, J. Amer. Math. Soc. 13 (2000), 611-637.

[St] B. Sturmfels, Gröbner Bases and Convex Polytopes, American Mathematical Society, Providence, RI, 1995.

[ST] B. Sturmfels and R. R. Thomas, Variation of cost functions in integer programming, Math. Programming 77 (1997), 357-387.

Received June 8, 2000, and in revised form November 2, 2000, April 11, 2001, and September 10, 2001.

Online publication April 19, 2002. 